\section{Buchrezension zu: Wechselwirkungen zwischen Arzneimitteln und Lebensmitteln}

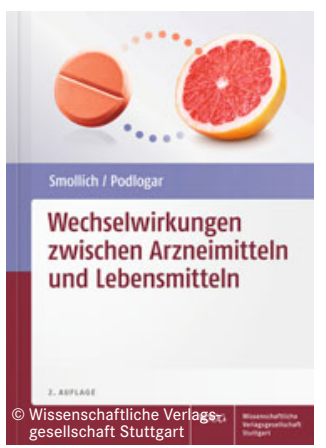

Wechselwirkungen

zwischen Arzneimitteln und

Lebensmitteln

Martin Smollich und Julia

Podlogar

133 S., 2. Aufl., Wissenschaftliche Verlagsgesellschaft Stuttgart, 2020. $S C, 29,80 €$

ISBN: 019783804740020

DOI: $10.1007 / \mathrm{s} 12268-021-1583-7$

(C) Der Autor 2021

Aus Erfahrung als langjähriger Dozent für das Fach Pharmakologie kann der Rezensent festhalten, dass das Thema des Buchs von Smollich und Podlogar von großem Interesse für Medizinund Pharmaziestudenten und natürlich Ärzte sowie Apotheker ist. In den gängigen Lehrbüchern der Pharmakologie und Toxikologie findet man leider keine systematische Abhandlung über den Zusammenhang zwischen Arzneitherapie und Lebensmitteln. Nur hier und da werden an verschiedenen Stellen Zusammenhänge aufgezeigt. Diese wichtige inhaltliche Lücke schließt das vorliegende, sehr anschaulich und kompakt geschriebene Buch von zwei Experten.

Zunächst werden Faktoren von Seiten des Arzneimittels, der Nahrung und des Patienten besprochen, die Interaktionen verursachen können, anschließend der Einfluss von Arzneimitteln auf Appetit, Geschmackssinn und Körpergewicht.

Im Haupteil des Buchs erfahren wir sehr anschaulich, wie Grape- fruitsaft, Milchprodukte, Alkohol, tyraminreiche Nahrungsmittel, Gojibeeren, Tee, Kaffee, VitaminK-haltige Gemüsesorten, Lakritze, Rotschimmelreis und Sojaprodukte mit der Wirkung von Arzneimitteln verstärkend oder abschwächend interferieren können. Teilweise können lebensbedrohliche Symptome auftreten. Auch die Histaminvergiftung durch Nahrungsmittel wird besprochen. Hilfreich sind die zahlreichen Tabellen und Abbildungen, Merksätze (bzw. Warnsätze) und Zusammenfassungen.

Die sehr anschaulichen und mit einprägsamen Piktogrammen versehenen Übersichten auf den Einbandseiten sind für die rasche Orientierung besonders wertvoll. Dort werden die wichtigsten Interaktionen von Arzneistoffen mit Nahrungsmitteln zusammengefasst und auf die entsprechenden Kapitel für weitere Einzelheiten verwiesen. Sehr hilfreich sind auch die Übersichtstabelle mit den 10 wichtigsten Interaktionen, das aktuelle Literaturverzeichnis, das sowohl deutsch- als auch englischsprachige Literatur umfasst, sowie ein Stichwortverzeichnis.

Der Rezensent kann festhalten, dass dieses Buch rundherum gelungen ist. Man kann es locker an zwei bis drei Abenden lesen. Der einzige Wermutstropfen ist der Preis: $29,80 €$ für 133 Seiten kartoniertes Buch ist doch eine Stange Geld. Das ist schade, da ein niedrigerer Preis für die Verbreitung dieses wichtigen Themas sehr förderlich wäre.

Roland Seifert,

Medizinische Hochschule Hannover, seifert.roland@mh-hannover.de Diese Rezension erscheint Open Access. *
* Funding note: Open Access funding enabled and organized by Projekt DEAL. Open Access: Dieser Artikel wird unter der Creative Commons Namensnennung 4.0 International Lizenz veröffentlicht, welche die Nutzung, Vervielfältigung, Bearbeitung, Verbreitung und Wiedergabe in jeglichem Medium und Format erlaubt, sofern Sie den/die ursprünglichen Autor(en) und die Quelle ordnungsgemäß nennen, einen Link zur Creative Commons Lizenz beifügen und angeben, ob Änderungen vorgenommen wurden. Die in diesem Artikel enthaltenen Bilder und sonstiges Drittmaterial unterliegen ebenfalls der genannten Creative Commons Lizenz, sofern sich aus der Abbildungslegende nichts anderes ergibt. Sofern das betreffende Material nicht unter der genannten Creative Commons Lizenz steht und die betreffende Handlung nicht nach gesetzlichen Vorschriften erlaubt ist, ist für die oben aufgeführten Weiterverwendungen des Materials die Einwilligung des jeweiligen Rechteinhabers einzuholen. Weitere Details zur Lizenz entnehmen Sie bitte der Lizenzinformation auf

http://creativecommons.org/licenses/ by $/ 4.0 /$ deed.de. 